\title{
USING SENSOR WEB PROCESSES AND PROTOCOLS TO ASSIMILATE SATELLITE DATA INTO A FORECAST MODEL
}

\author{
H. Michael Goodman', Helen Conover', Bradley Zavodsky', Manil Maskey', Gary Jedlovec', Kathryn \\ Regner ${ }^{2}$, Xiang Li ${ }^{2}$, Jessica Lu ${ }^{2}$, Mike Botts ${ }^{2}$, and Gregoire Berthiau ${ }^{2}$

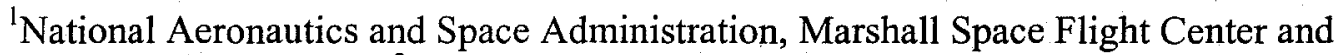 \\ ${ }^{2}$ University of Alabama-Huntsville
}

\section{INTRODUCTION}

The goal of the Sensor Management Applied Research Technologies (SMART) On-Demand Modeling project is to develop and demonstrate the readiness of the Open Geospatial Consortium (OGC) Sensor Web Enablement (SWE) capabilities to integrate both space-based Earth observations and forecast model output into new data acquisition and assimilation strategies. The project is developing sensor web-enabled processing plans to assimilate Atmospheric Infrared Sounding (AIRS) satellite temperature and moisture retrievals into a regional Weather Research and Forecast (WRF) model over the southeastern United States.

\section{AIRS DATA ASSIMILATION INTO WRF MODEL}

Our initial efforts in the SMART project focused on applying SWE protocols and encodings to atmospheric satellite data coincidence tools and analyses. We implemented a satellite coincidence tool that utilized many SWE elements for data access and transport. The Cloud Intersection and Validation Tool demonstrated the utility of using Sensor Model Language (SensorML) tools and Sensor Observation Service (SOS) interface protocols to detect the coincidence of CloudSat observations with Geostationary Operational Environmental Satellite (GOES) Cloud Top Pressure values bounded by date, time, location, and atmospheric pressure range.

Our current focus is on demonstrating the use of sensor web standards in the assimilation of AIRS satellite data into WRF forecast model runs. Using knowledge gained from the implementation of a variety of SWE technologies in our Cloud Intersection and Validation demonstration testbed, as well as tutorials on additional SWE technologies, the SMART team has completed definition of our AIRS data assimilation scenario for use in weather forecast models. Figure 1 shows the three primary functions required for this scenario: data access, event identification, and data assimilation. In this scenario, operational forecasts from the North American Model (NAM) and observations from the AIRS instrument aboard the Aqua satellite are acquired as they are generated several times per day, and made available via SOS services. The NAM forecasts parameters are mined for appropriate weather phenomena (e.g., precipitation, cold frontal location, low pressure centers, etc., see figure 1, step 1) and a Sensor Alert Service will issue a notification whenever AIRS observations are coincident with these weather events (figure 1, step 2). In response to such an alert, a SensorML Process Chain will acquire NAM forecasts parameters along with AIRS observations of the phenomena detected, and will initiate the data assimilation process (figure 1, step 3). Finally, a Web Notification Service associated with the data assimilation process will issue an alert to WRF modelers when the subsetted data assimilation set is complete and a new set of initial conditions for the WRF model is available (figure 1 , step 4). 


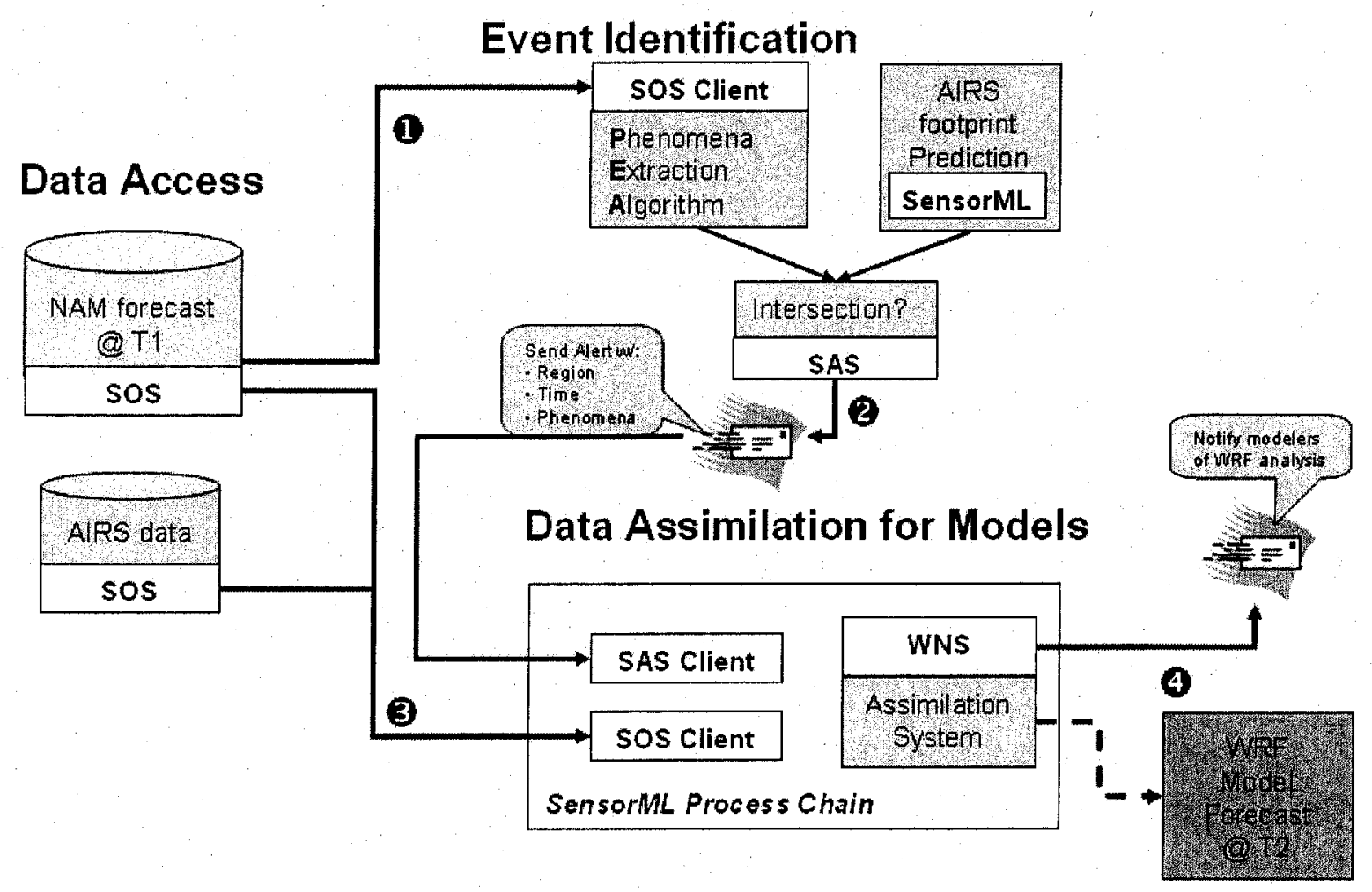

Figure 1: SMART assimilation of AIRS data into weather forecast models

The initial implementation of the science demonstration is accessing historical NAM forecast runs and archived AIRS data. Subsequent versions will culminate with the system prototype of near real-time satellite assimilation into the WRF model.

The success of SWE in applied science systems will only be achieved through the proliferation of SWE technologies within the science community. By applying SWE protocols to a real world science exercise we are demonstrating the utility and promise of a more timely and efficient satellite data assimilation process. 